\title{
Effect of strain rate on the fracture behaviour of epoxy-graphene nanocomposite
}

\author{
RAHIM EQRA ${ }^{1,2, *}$ and MOHAMMAD HADI MOGHIM ${ }^{1}$ \\ ${ }^{1}$ Department of Materials Science and Engineering, Engineering School, Shiraz University, Shiraz 71348-15939, Iran \\ ${ }^{2}$ Institute of Mechanics, ISRC, Shiraz 71555-414, Iran
}

MS received 9 October 2015; accepted 17 March 2016

\begin{abstract}
In this study, epoxy-based nanocomposite was fabricated by the addition of graphene nanosheet via a solution casting method. To investigate the effect of strain rate on tensile properties of epoxy, tensile tests were done on standard samples at different strain rates $\left(0.05-1 \mathrm{~min}^{-1}\right)$. The role of strain rate and presence of graphene on fracture behaviour of epoxy were also studied by investigation of the fracture surfaces of some samples by scanning electron microscopy (SEM). Finally, Eyring's model was performed to clarify the role of strain rate on activation volume and activation enthalpy of epoxy. The results of tensile tests showed a maximum strength of epoxy-graphene nanocomposite at the graphene wt \% of $0.1 \%$. Tensile strength of epoxy obviously improved with increasing strain rate, but tensile strength of epoxy/graphene nanocomposite sample was less sensitive. Fracture micrographs showed that the mirror zone of the fracture surface of epoxy diminished by increasing strain rate or addition of graphene; and final fracture zone also became rougher. Finally, by investigation of the activation enthalpies, it was showed that much higher enthalpy was needed to fracture the nanocomposite sample, as the activation enthalpy changed from 41.54 for neat epoxy to $67.34 \mathrm{~kJ} \mathrm{~mol}^{-1}$ for EP-0.1\% GNS sample.
\end{abstract}

Keywords. Epoxy; graphene; strain rate; fracture behaviour; nanocomposite.

\section{Introduction}

In many engineering applications, materials are loaded at different strain rates, and the rate of loading has been known to affect the mechanical behaviour of polymers quite significantly. Therefore, a deep understanding about the effect of strain rate on polymer properties is crucial in the design and safe in service performance of polymeric structural components [1]. Almost all different mechanical properties of polymers, such as elastic modulus, yield and strength are sensitive to the rate of loading and deformation [2].

Also, it is proved that the properties of polymeric materials are enhanced by the addition of nanofiller, without compromising their process ability, inherent mechanical properties and light weights [3]. Polymer-based nanocomposite materials have attracted significant interests in the fields of material science and engineering, for the purpose of creating new materials with new or enhanced properties compared with their pristine, individual material [4]. Graphene has been considered extensively, since its discovery by Geim and colleagues [5] in 2004. Graphene nanosheet (GNS) is an atomically thick, two-dimensional (2-D) sheet material composed of $\mathrm{sp}^{2}$ carbon atoms arranged in a honeycomb structure [6,7]. This carbon-based nanofiller has been attracted as a promising candidate to create new polymer matrix nanocomposites due to its excellent properties and ready availability of its

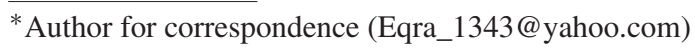

precursor, graphite. The incorporation of graphene can dramatically enhance the electrical, physical, mechanical and barrier properties of polymer composites at extremely low loadings [8,9].

On the other hand, epoxy (EP) resins are a class of high overall performance thermosetting polymers that have wide applications as the main component for adhesives and matrices for composites, etc. in various industries [10]. This family presents good stiffness, specific strength, dimensional stability, chemical resistance and strong adhesion to the reinforcement [11]. Therefore epoxies are used as the most common matrix in the polymer nanocomposites. Figure 1 shows a schematic picture of epoxy-graphene nanocomposite.

The effects of strain rate on pure polymers and composites have been studied by several researchers. The mechanical properties of most of these materials are reported to be rate-sensitive [12]. Jacob et al [13] have provided a nice review on works conducted to investigate the strain rate dependency of mechanical properties of polymer composite materials. Moghim and Zebarjad [2] investigated the effect of carbon nanotubes (CNTs) on the mechanical properties of polyurethane-based nanocomposites. They observed a positive intense effect of strain rate on tensile properties of polyurethane/multiwalled carbon nanotubes (PU/MWCNTs) nanocomposites and also proved that this dependency decreased as CNTs content increased. Guo and Li [14] studied the quasi-static and dynamic characteristics of epoxy- $\mathrm{SiO}_{2}$ nanocomposites under uniaxial compression at different loading rates $\left(10^{-4}-10^{4} \mathrm{~s}^{-1}\right)$. They found that the 

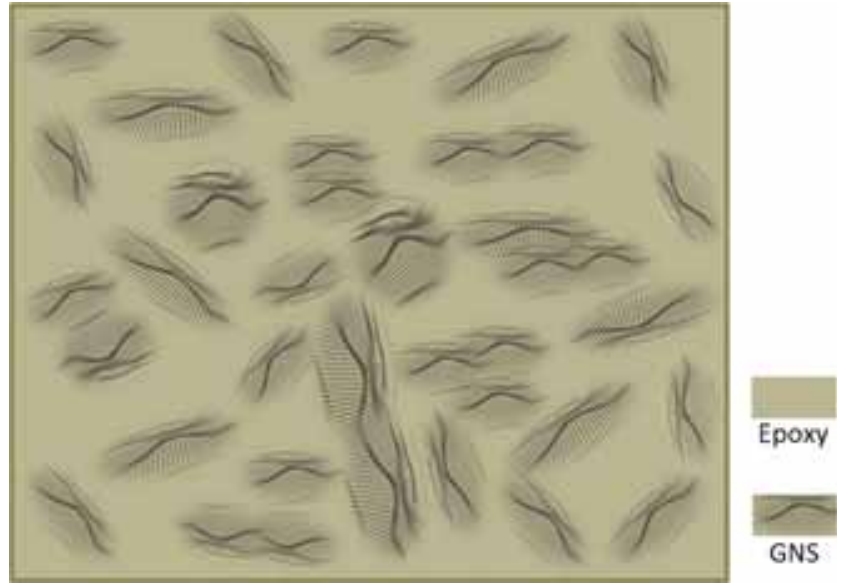

Figure 1. Schematic picture of epoxy-graphene nanocomposite.

compressive strength of the composites is higher than that of pure epoxy at high strain-rates. Saniee et al [15] investigated the rate dependency of glass/epoxy composites at low strain rates. They observed that the longitudinal strength has increased to $24.7 \%$ by increasing the strain rate from 0.0001 to $0.11 \mathrm{~s}^{-1}$. Ma et al [11] worked on the compression behaviours of epoxy with CNTs under high strain rates. Their results indicated that the stress-strain curves were strain-rate sensitive, and the compressive stiffness and compressive failure stress of composites with various CNT contents were increased with the strain rates and CNT contents. Shadlou et al [1] studied the effect of strain-rate on the mechanical behaviour of graphene reinforced epoxy/nanocomposites. They showed that the yield strength and Young's modulus of both the neat epoxy and nanocomposites increased at higher strain rates. They assessed the predictive capability of some of the available models for evaluating the strength and modulus of nanocomposites and compared against the experimental data obtained.

Despite the existence of these researches, our literature survey revealed that very little research has been published regarding the effect of strain rate on fracture behaviour of epoxy-graphene nanocomposites. Hence, in the current research, the effect of strain rate on tensile properties and fracture morphology of epoxy in the presence of graphene was investigated. Finally, thermodynamic properties of yielding i.e., activation volume and activation energy were also calculated using Eyring's model.

\section{Experimental}

\subsection{Materials and methods}

Epoxy resin (M506) based on the bisphenol-A and hardner (HA11) polyamine were supplied by Mokarrar Engineering Materials, Inc. (Tehran, Iran). The graphene nanosheets were fabricated by thermal expansion and reduction method of graphite in our previous work [3]. Figure 2 shows the

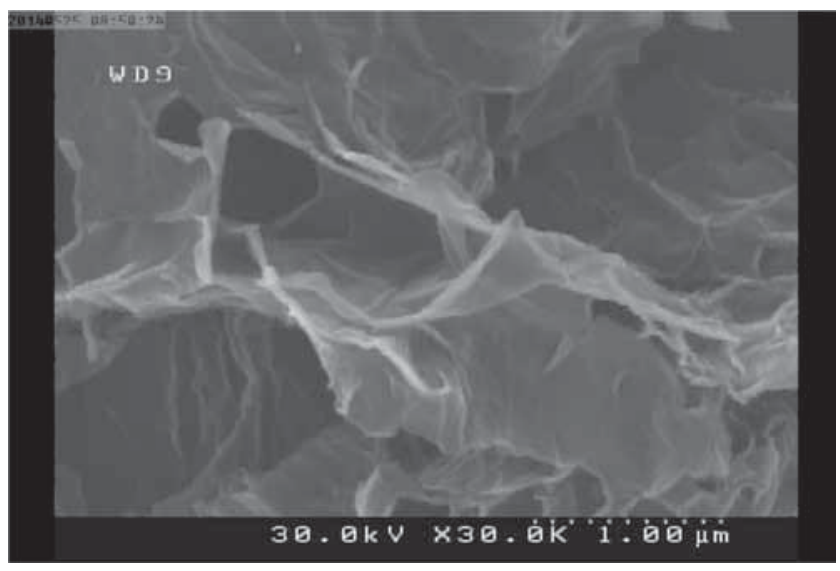

Figure 2. SEM of graphene.

scanning electron micrograph (SEM) of graphene which was used in this study.

Epoxy-graphene nanocomposites with different contents of graphene (i.e., $0.1-2.0 \mathrm{wt} \%$ ) were fabricated through the solution casting method. The produced GNS was agitated using an ultrasonic instrument under $100 \mathrm{~W}$ in acetone for $2 \mathrm{~h}$. Then, epoxy resin was added to the solution and sonicated under above condition for $90 \mathrm{~min}$. The mixture was heated under vacuum at $60^{\circ} \mathrm{C}$ for $18 \mathrm{~h}$ to remove acetone. Afterwards, hardner was added and mixed by a magnetic stirrer. This mixture was degassed in vacuum chamber and was injected into the tensile samples mould based on ASTM D638.

A Fourier-transform infrared (FTIR) spectrometer (Bruker, Tensor 27) was used to evaluate the interfacial interaction between GNS and epoxy matrix. The GNS or nanocomposite sample powder mixture with the $\mathrm{KBr}$ powder was moulded into discs for this characterization. Tensile test of the nanocomposite samples was done under a $100 \mathrm{kN}$ load cell with different strain rates $\left(0.05-1 \mathrm{~min}^{-1}\right)$ on three samples for each percentage. To clarify the effect of strain rate on the fracture behaviour of epoxy and EP/GNS nanocomposites, the fracture surfaces of some samples were studied using SEM (Leica Cambridge S360). It will be mentioned later that to investigate the effect of strain rate and exclude the effect of agglomeration on the tensile properties of epoxy graphene nanocomposite, it was possible to study two pure epoxy and epoxy- $0.1 \%$ GNS samples.

\subsection{Eyring model}

Thermodynamic properties of yielding were obtained in this study using Eyring's model. The well-known Eyring rate expression is the way of investigating the effect of strain rate on the flow stress of polymers, which seeks to do this from a molecular model of flow mechanism. The fundamental basis is that a segment of macromolecule must pass over an energy barrier, moving from one position to another in the solid polymer. In the absence of stress, the segments of polymer jump over the barrier very infrequently and they do so in random directions. It should be considered that in the Eyring's 
model, it is assumed that applied stress adjusts this energy barrier. It should also be mentioned that the stress required to cause yielding depends on this energy barrier [2].

The final equation of the Eyring model which used for investigating the effect of strain rate on yielding stress is as follows [16]:

$$
\left(\frac{\sigma_{\mathrm{y}}}{T}\right)=\left(\frac{2}{V^{*}}\right)\left[\left(\frac{\Delta H}{T}\right)+2.303 R \log \left(\frac{\dot{\varepsilon}_{\mathrm{y}}}{\dot{\varepsilon}_{0}}\right)\right] .
$$

In this equation, $\sigma_{\mathrm{y}}$ is the yielding stress, $T$ the absolute temperature, $V^{*}$ the activation volume, $\Delta H$ the activation enthalpy, $\dot{\varepsilon}_{\mathrm{y}}$ the strain rate at yielding point, $\dot{\varepsilon}_{0}$ a constant and $R$ the universal gas constant. By using this equation, $\sigma_{\mathrm{y}} / T$ $v s$. strain rate, linear equation can be obtained as

$$
\frac{d\left(\sigma_{\mathrm{y}} / T\right)}{d \log \dot{\varepsilon}_{\mathrm{y}}}=\frac{2 \times 2.303 \times R}{V^{*}} .
$$

Activation volume and activation enthalpy of different samples were the parameters which were calculated by using these equations.

\section{Results and discussion}

\subsection{FTIR spectra}

To investigate the nature of interactions between the chemical groups of epoxy and graphene, infrared spectra is usually used. Figure 3 shows the infrared spectra of GNS, epoxy and epoxy-graphene nanocomposite. The broad band around $3300 \mathrm{~cm}^{-1}$ is related to the stretching vibration of hydroxyl groups (O-H) of epoxy and GNS. Peaks at 2925 and $2855 \mathrm{~cm}^{-1}$ are attributed to the stretching vibration groups (C-H), respectively in $\mathrm{CH}_{2}$ and $\mathrm{CH}_{3}$ bands of epoxy. Peaks at 1726,1226 and $1103 \mathrm{~cm}^{-1}$ are also in the range of stretching vibration bands of carbonyl $(\mathrm{C}=\mathrm{O})$ and carboxyl $(\mathrm{C}-\mathrm{OH})$ and epoxide $(\mathrm{C}-\mathrm{O})$ groups, respectively. Finally, peaks at 1050,1440 and $1625 \mathrm{~cm}^{-1}$ are attributed to $\mathrm{C}-\mathrm{O}$ stretching vibrations of the epoxide groups, $\mathrm{C}-\mathrm{OH}$ stretching vibrations of carboxyl groups, $\mathrm{C}=\mathrm{O}$ stretching vibrations from carbonyl groups of GNS $[17,18]$.

\subsection{Tensile strength of EP-GNS nanocomposites}

The dependence of tensile strength of epoxy on graphene content is shown in figure 4. As seen, strength obviously
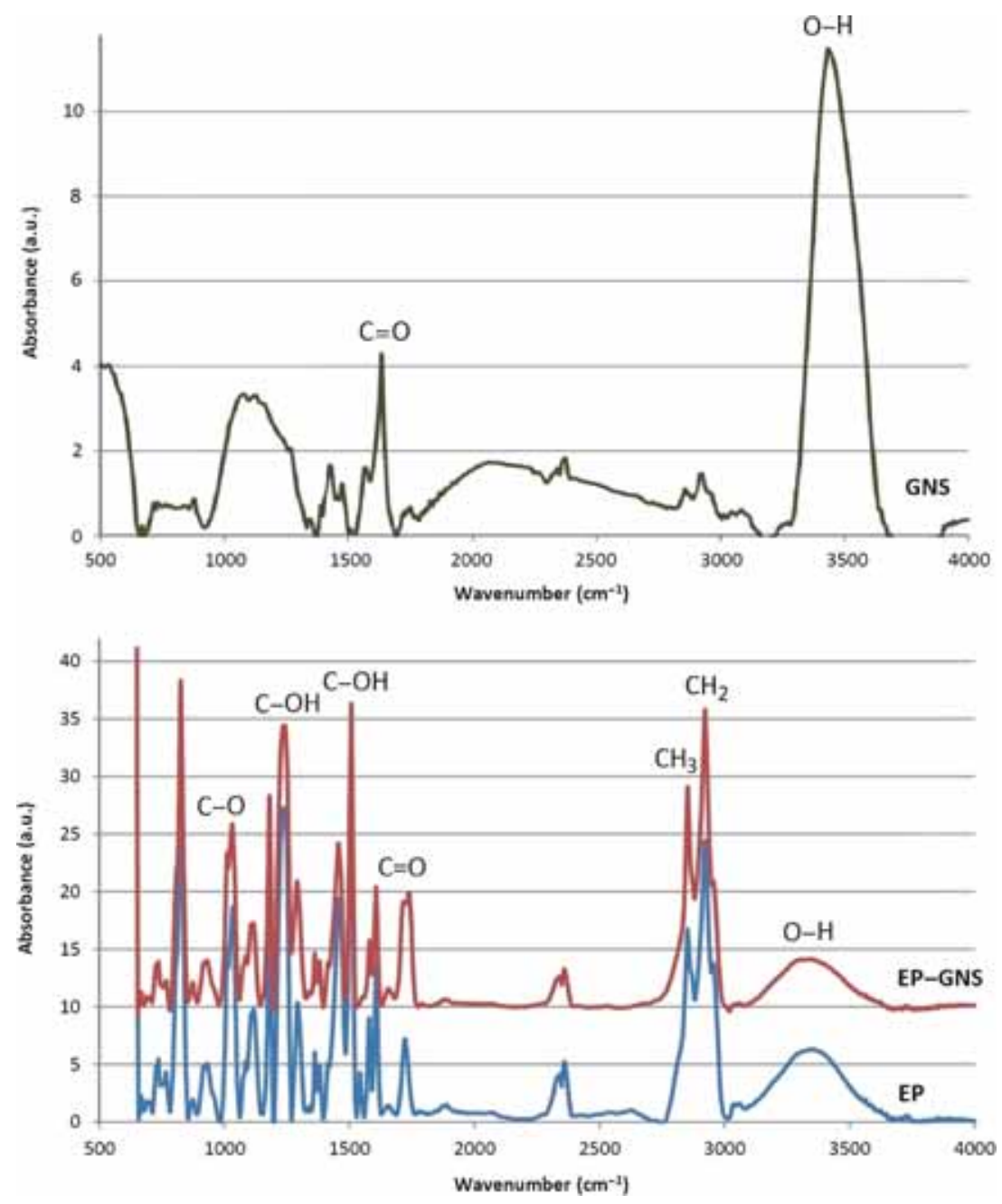

Figure 3. Infrared spectra of GNS, epoxy and EP-0.1 wt\% GNS nanocomposite. 


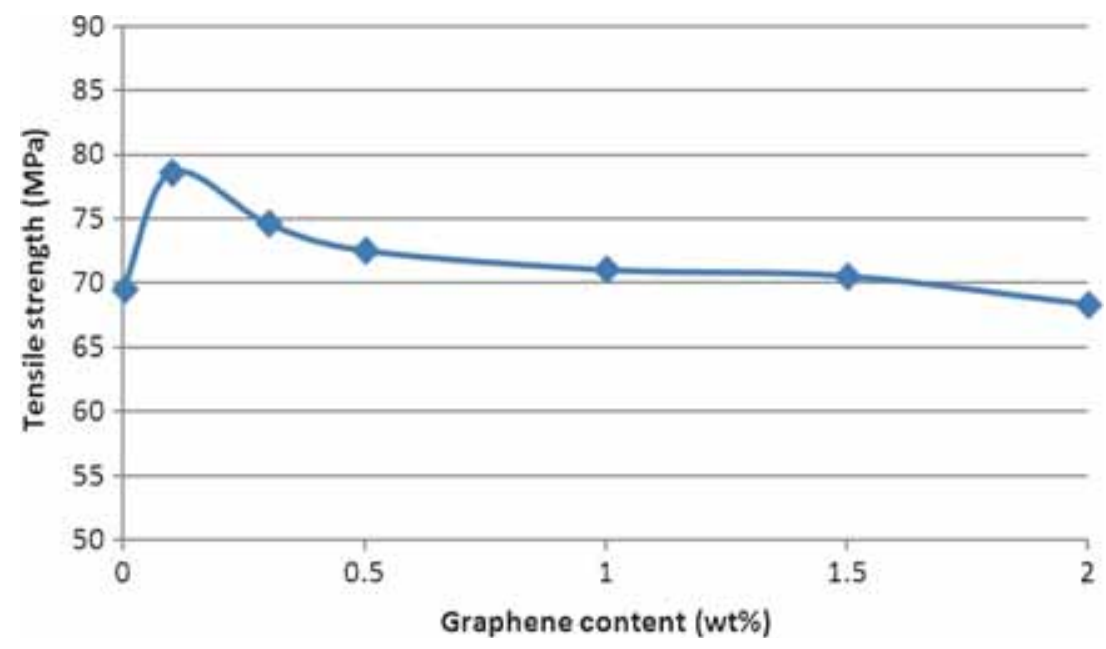

Figure 4. Tensile strength curve for neat epoxy and its nanocomposites with different graphene loadings.

improves in comparison with neat epoxy by the incorporation of GNS into the EP matrix and this continues up to $0.1 \mathrm{wt} \%$. Then, an inverse effect is observed and mechanical properties decline with the addition of more graphene. The strength of EP-0.1\% GNS is $13 \%$ higher than epoxy. The same results are reported by other researchers $[19,20]$. As a fact, at low contents of graphene, reinforcing effect is observed due to a good dispersion. But at higher contents, strength drops sharply by the crack initiation in the agglomerated regions of GNS. These agglomerations act as defect regions which serve to initiate fracture. Moreover, tensile strength's dependence of nanocomposites to the stress transfer between rigid fillers and matrix may be responsible for later effect. If the interface between nanofiller and matrix was sufficiently strong, the stress would be transferred to the reinforcements successfully and in this case, the strength of composite is high. But addition of more reinforcement results in reduction of strength, because of weak interfacial bonding. Therefore, neat epoxy and EP- $0.1 \%$ GNS samples were selected for the study of the effect of strain rate to remove the effect of agglomeration.

\subsection{Tensile stress-strain curve at different strain rates}

The effect of strain rate on tensile behaviour of EP and EP- $0.1 \%$ GNS is shown in figure 5. As seen, tensile properties of epoxy are affected by strain rate, but the dependency is reduced by the incorporation of the GNS. The elongations at break are comparable, but elastic modulus of the samples is enhanced by the strain rate. Moreover, tensile strength of samples improves obviously with strain rate. The dependence of tensile strength of epoxy on strain rate and GNS content is shown in figure 6. As seen, tensile strength of epoxy is much strain-rate sensitive than epoxy/graphene nanocomposite. As a fact, tensile strength of epoxy and EP-0.1\% GNS increases by $21 \%$ (14.09 MPa) and $15 \%(10.8 \mathrm{MPa})$, respectively, with the change of strain rate from 0.05 to $1 \mathrm{~min}^{-1}$. This lower strain rate sensitivity can be attributed to the confinement which is imposed on the molecular mobility and intermolecular interaction of epoxy molecular chains by the presence of graphene nanosheets [2]. The GNP particles with their higher stiffness (i.e., compared to neat epoxy) get located within the spaces existing among the polymer chains, thus, reducing chain's flexibility. They also act as obstacles to the changes in intermolecular configuration.

\subsection{Fracture behaviour at different strain rates}

To investigate the fracture behaviour of epoxy microscopically, fracture surface of some samples were studied. Generally, the fracture surface of epoxy contains three zones that are shown in figure 7 . The smooth or mirror zone that forms in the vicinity of initiation of initial crack, the transition zone in which roughness uniformly increases and the final fracture zone that has nonuniform rough surface and is rapidly formed in the final stage of deformation. The morphology of these zones is affected by the growth rate of the initial crack and the initiation rate of the secondary cracks in tip of the initial cracks [21]. Thus, the extent and existence of these zones depend on factors that control theses rates, such as strength, toughness and characteristic of fillers in the epoxy matrix. As seen in figure 7 , mirror zone of the fracture surface of epoxy was diminished by increasing the strain rate. In fact, at higher strain rate $\left(0.5 \mathrm{~min}^{-1}\right)$ for epoxy (figure 7b) and EP-GNS nanocomposite (figure 7d), smaller mirror zone is observed than the micrographs of epoxy (figure 7a) and EP-GNS nanocomposite (figure 7c) at lower strain rate $\left(0.05 \mathrm{~min}^{-1}\right)$. The same behaviour is also appeared with the addition of graphene and the mirror zone converted to rough surface. This phenomenon is revealed in both the strain rates. As a fact, addition of GNS diminished mirror zone of the microstructure of epoxy at the two strain rates of $0.05 \mathrm{~min}^{-1}$ (figure $7 \mathrm{c}$ with respect to figure $7 \mathrm{a}$ ) and $0.5 \mathrm{~min}^{-1}$ (figure $7 \mathrm{~d}$ with respect to figure $7 \mathrm{~b}$ ). It can be due 

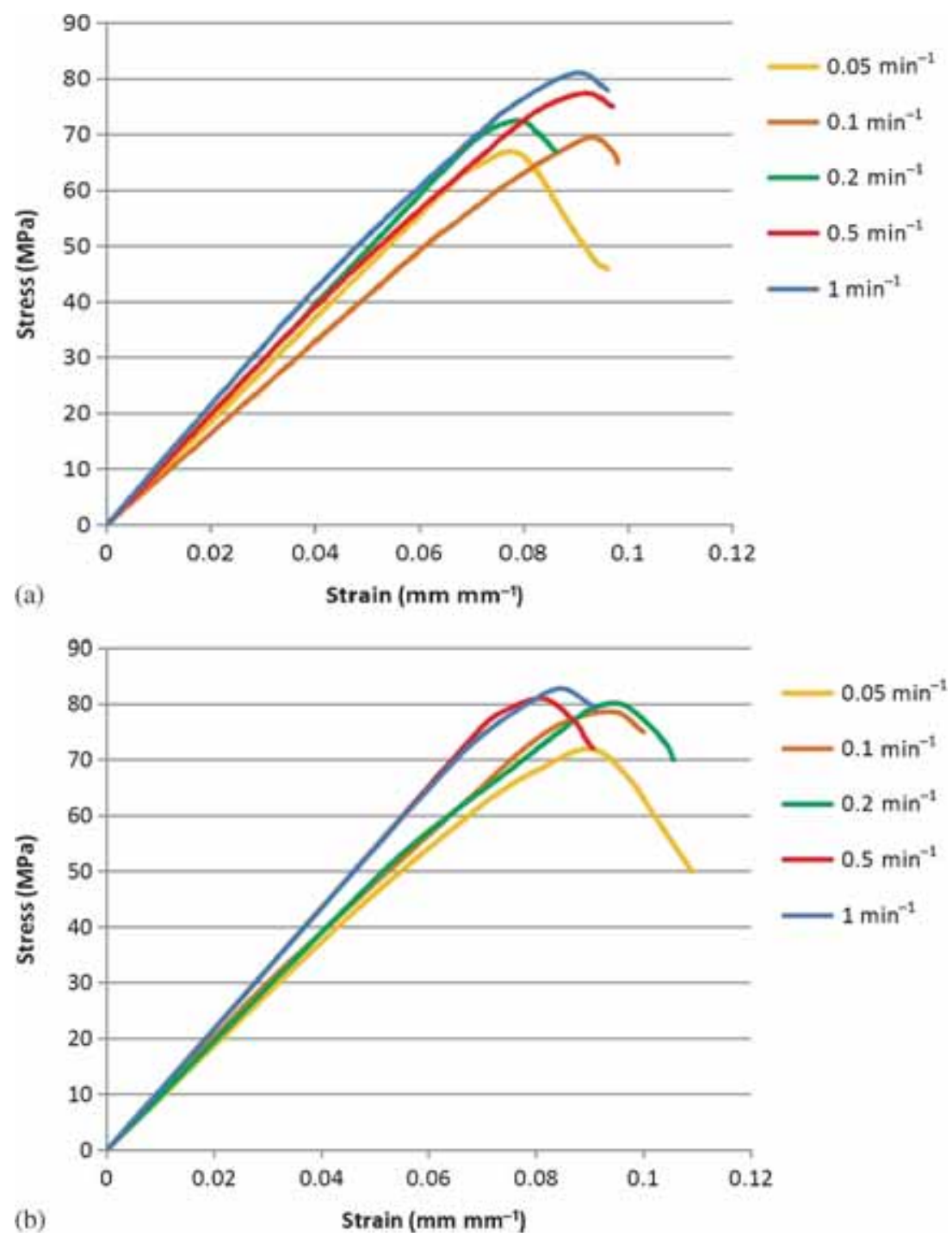

Figure 5. Effect of strain rate on tensile stress-strain behaviour of (a) neat epoxy and (b) EP-0.1\% GNS.

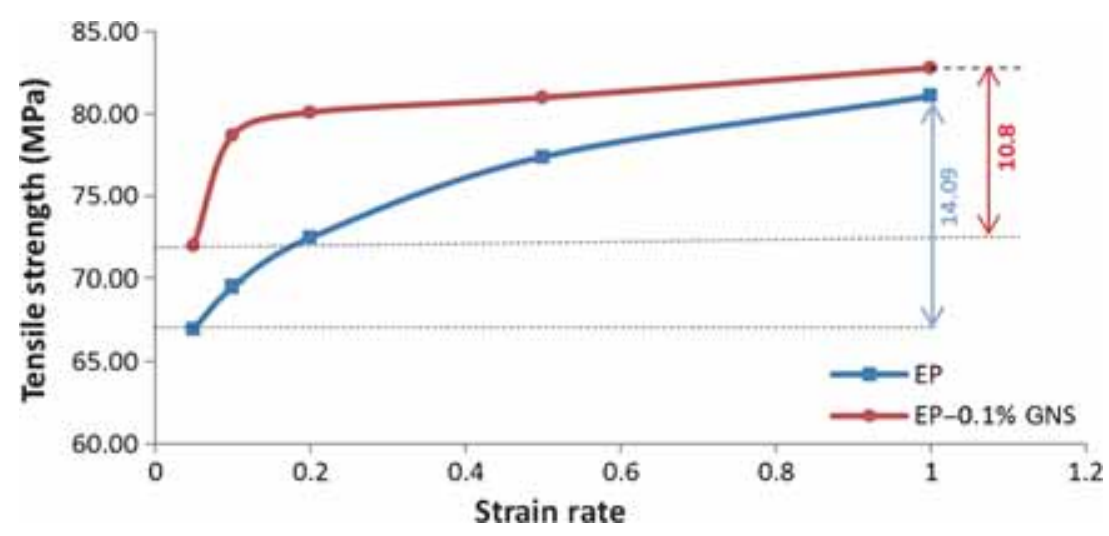

Figure 6. Effect of strain rate on the strength of epoxy and EP-0.1\% GNS.

to the higher strength and stiffness of epoxy in the presence of graphene at higher strain rate, which caused secondary cracks to be formed immediately.
Moreover, other rough zones (transition and final fracture zone) became rougher at higher strain rates and in the presence of GNS. This can be seen by studying the morphology 

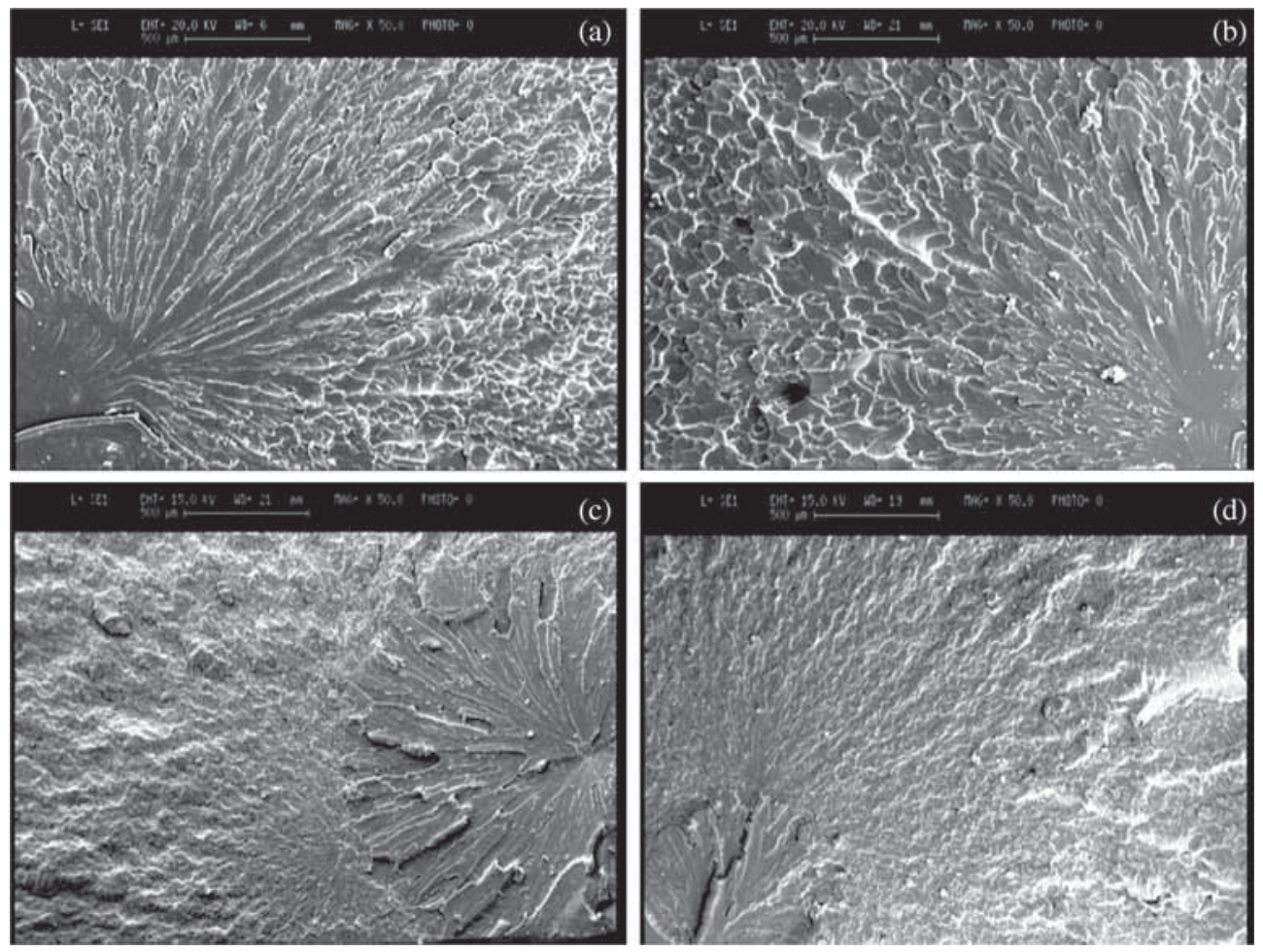

Figure 7. SEM micrographs of the fracture surfaces of $(\mathbf{a}, \mathbf{b})$ EP and (c,d) EP-0.1\% GNS; at two different strain rates of 0.05 and $0.5 \mathrm{~min}^{-1}$.
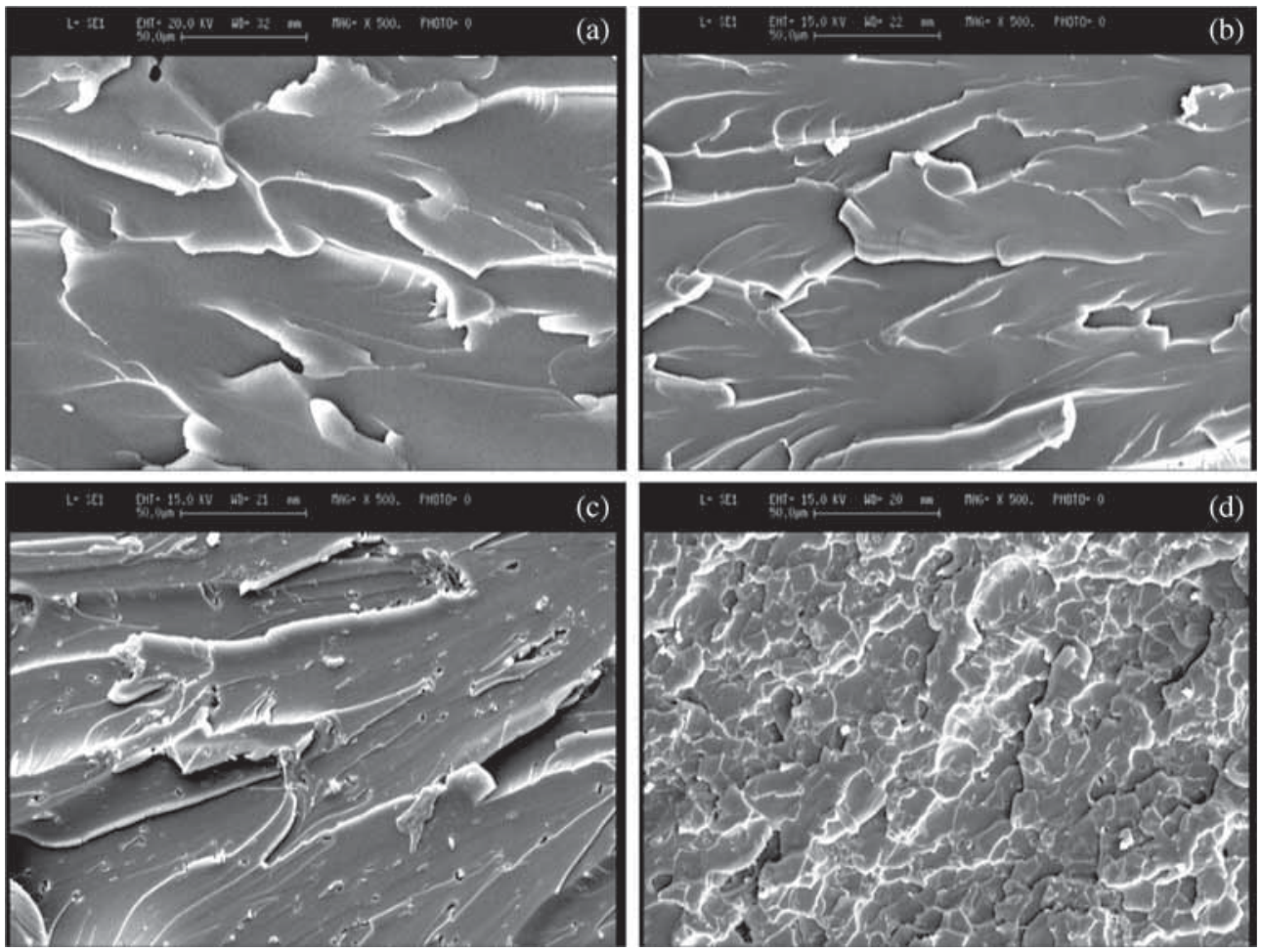

Figure 8. SEM micrographs of the final stage in fracture surfaces of $(\mathbf{a}, \mathbf{b})$ EP and $(\mathbf{c}, \mathbf{d})$ EP- $0.1 \%$ GNS; at two different strain rates of 0.05 and $0.5 \mathrm{~min}^{-1}$.

of the final stage in fracture surface of epoxy (figure 8). The enhancement of roughness is generally attributed to crack path deflection mechanism which introduced by graphene in the epoxy matrix. However, these zones are still recognizable with smaller transition zones and larger uniform final fracture zone, which can be attributed to higher 


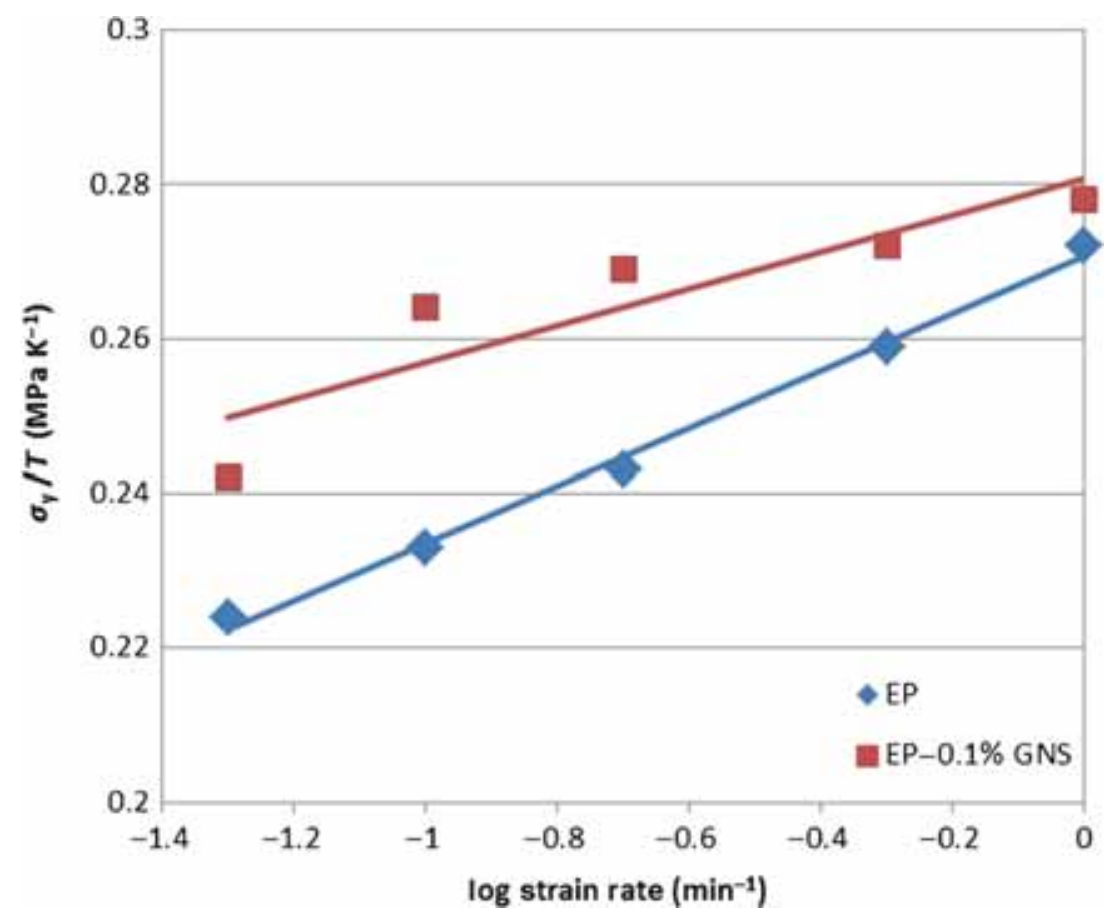

Figure 9. $\left(\sigma_{\mathrm{y}} / T\right)$ vs. $\log$ strain rate for EP and EP-0.1\% GNS.

Table 1. Linear equations of $\left(\sigma_{\mathrm{y}} / T\right) v s$. $\log$ strain rate for EP and EP-0.1\% GNS.

\begin{tabular}{lc}
\hline Samples & Linear equation \\
\hline EP & $Y=0.0371 X+0.2707$ \\
EP-0.1\% GNS & $Y=0.0238 X+0.2807$ \\
\hline
\end{tabular}

strength and toughness of the 0.1 wt $\%$ graphene-epoxy nanocomposite.

\subsection{Eyring model}

To investigate the fracture behaviour of epoxy with and without the incorporation of GNS more precisely, molecular mechanism of fracture should be studied. In this regard, wellknown Eyring rate expression is employed here to obtain the activation enthalpy of fracture. Figure 9 shows the diagram of $\left(\sigma_{\mathrm{y}} / T\right) v s$. log strain rate for EP and EP-0.1\% GNS. As shown, this figure shows the linear relationship between $\left(\sigma_{\mathrm{y}} / T\right)$ and $\log$ strain rate which confirms that Eyring model can be applicable here. Table 1 shows the linear equations of $\left(\sigma_{\mathrm{y}} / T\right)$ vs. $\log$ strain rate for EP and EP-0.1\% GNS. By comparing the slope and $Y$-intercept of these lines with the equations of Eyring model, activation volume and activation enthalpy of EP and EP-0.1\% GNS were obtained (table 2).

The calculated activation enthalpies are in the range and comparable to the literature data $[1,22]$. As expected, it
Table 2. Activation volume and activation enthalpy of EP and EP- $0.1 \%$ GNS.

\begin{tabular}{lcc}
\hline Samples & $\begin{array}{c}\text { Activation volume } \\
\left(\mathrm{m}^{3} \mathrm{~mol}^{-1}\right)\end{array}$ & $\begin{array}{c}\text { Activation enthalpy } \\
\left(\mathrm{kJ} \mathrm{mol}^{-1}\right)\end{array}$ \\
\hline EP & $1.03 \times 10^{-3}$ & 41.54 \\
EP-0.1\% GNS & $1.61 \times 10^{-3}$ & 67.34 \\
\hline
\end{tabular}

needs much activation enthalpy for the composite samples, as the activation enthalpy changes from 41.54 for neat epoxy to $67.34 \mathrm{~kJ} \mathrm{~mol}^{-1}$ for EP- $0.1 \%$ GNS sample, which is because of the higher strength of nanocomposite samples. The increase in the mechanical properties of nanocomposite sample is because of the reduction in molecular mobility of epoxy chains, which results in stiffer chains. In fact, it needs more energy for the mobility of molecules with the addition of graphene nanosheets.

\section{Conclusion}

In this research, epoxy/graphene nanocomposite was fabricated through the solution casting method. To investigate the effect of strain rate on tensile properties of epoxy, tensile tests were conducted at different strain rates $\left(0.05-1 \mathrm{~min}^{-1}\right)$; and the fracture surface of epoxy was studied by SEM. Eyring's model was also used to confirm the role of strain rate on the mechanical properties of EP-GNS nanocomposite. The result of tensile tests showed that EP-0.1\% GNS had maximum strength and the strength of neat epoxy and epoxy 
graphene nanocomposite were enhanced with increase in strain rate, but tensile strength of epoxy/graphene nanocomposite sample was less sensitive. Investigating the fracture surface of different samples showed that the mirror zone of the fracture surface of epoxy diminishes by increasing the strain rate or addition of graphene and final fracture zone also became rougher. Finally, by investigation of the activation enthalpies, it was showed that much higher enthalpy is needed to fracture the nanocomposite sample, as the activation enthalpy changes from 41.54 for neat epoxy to $67.34 \mathrm{~kJ} \mathrm{~mol}^{-1}$ for Ep-0.1\% GNS sample.

\section{References}

[1] Shadlou S, Ahmadi-Moghadam B and Taheri F 2014 Mater. Des. 59439

[2] Moghim M H and Zebarjad S M J. Vinyl Addit. Techn. in press

[3] Eqra R, Janghorban K and Daneshmanesh H 2015 J. Polym. Eng. 35257

[4] Tijing L D, Park C H, Choi W L, Ruelo M T G, Amarjargal A, Pant H R et al 2013 Compos. Part B 44613

[5] Navoselov K S, Geim A K, Morozov S V, Jiang D, Zhang Y, Dubonos S V et al 2004 Science 306666

[6] Gong J R 2011 Graphene-synthesis, characterization, properties and applications (Croatia: InTech)

[7] Wajid A S, Das S, Irin F, Tanvir Ahmed H S, Shelburne J L, Parviz D et al 2012 Carbon 50526
[8] Galpaya D, Wang M, Liu M, Motta N, Waclawik E and Yan C 2012 Graphene 130

[9] Das T K and Prusty S 2013 Polym. Plast. Technol. Eng. 52319

[10] Shen X J, Liu Y, Xiao H M, Feng Q P, Yu Z Z and Fu S Y 2012 Compos. Sci. Technol. 721581

[11] Ma P, Jiang G, Chen Q, Cong H and Nie X 2015 Compos. Part B 69526

[12] Gurusideswar S and Velmurugan R 2014 Mater. Des. 60468

[13] Jacob G C, Starbuck J M, Fellers J F, Simunovic S and Boeman R G 2004 J. Appl. Polym. Sci. 94296

[14] Guo Y and Li L 2007 Mater. Sci. Eng. A 458330

[15] Saniee F F, Majzoobi G H and Bahrami M 2005 J. Mater. Process Technol. 162-163 39

[16] McCrum N G, Buckley C P and Bucknall C B 2011 Principles of polymer engineering (UK: Oxford University Press).

[17] Naebe M, Wang J, Amini A, Khayyam H, Hameed N, Li L et al 2014 Sci. Rep. 41

[18] Li L, Wu Q, Li S and Wu P 2008 Appl. Spectrosc. 621129

[19] Chatterjee S, Wang J W, Kuo W S, Tai N H, Salzmann C, Li W L et al 2012 Chem. Phys. Lett. 5316

[20] Wang X, Xing W, Zhang P, Song L, Yang H and Hu Y 2012 Compos. Sci. Technol. 72737

[21] Moraesd'Almeidaa J R and De Menezesb G W 2003 Mater. Res. 6415

[22] Gomez-Del Rio T, Salazar A and Rodriguez J 2012 Mater. Des. 42301 